ARTICLE

Received 18 Sep 2012 | Accepted 23 Oct 2012 | Published 20 Nov 2012

DOI: 10.1038/ncomms2215

\title{
Synthesis of chiral $\mathrm{TiO}_{2}$ nanofibre with electron transition-based optical activity
}

Shaohua Liu ${ }^{1, \star}$, Lu Han $^{1, \star}$, Yingying Duan ${ }^{1, \star}$, Shunsuke Asahina ${ }^{2}$, Osamu Terasaki ${ }^{3,4}$, Yuanyuan Cao ${ }^{1}$, Ben Liu ${ }^{1}$, Liguo Ma', Jialiang Zhang ${ }^{1} \&$ Shunai Che ${ }^{1}$

The optical chirality induced at the absorption bands due to electronic exciton coupling of the transition dipole moments between chromophores in close proximity is ubiquitous in helical organic materials. However, inorganic materials with optical activity resulting from electronic transitions have not been explored. Here we report the synthesis of chiral $\mathrm{TiO}_{2}$ fibres via transcription of the helical structure of amino acid-derived amphiphile fibres through coordination bonding interactions between the organics and the $\mathrm{TiO}_{2}$ source. Upon calcination, the as-prepared amorphous $\mathrm{TiO}_{2}$ double-helical fibres with a pitch length of $\sim 100 \mathrm{~nm}$ were converted to double-helical crystalline fibres with stacks of anatase nanocrystals in an epitaxial helical relationship. Both the amorphous and anatase crystalline helical $\mathrm{TiO}_{2}$ fibres exhibited optical response to circularly polarized light at the absorption edge around $\sim 350 \mathrm{~nm}$. This was attributed to the semiconductor $\mathrm{TiO}_{2}$-based electronic transitions from the valence band to the conduction band under an asymmetric electric field.

\footnotetext{
${ }^{1}$ School of Chemistry and Chemical Engineering, State Key Laboratory of Metal Matrix Composites, Shanghai Jiao Tong University, 800 Dongchuan Road, Shanghai 200240, China. ${ }^{2}$ SMBU, JEOL, Akishima, Tokyo 196-8558, Japan. ${ }^{3}$ Graduate School of EEWS, WCU, KAIST, Daejeon, Republic of Korea.

${ }^{4}$ Department of Materials and Environmental Chemistry, Bezelii Center EXSELENT on Porous Materials, Stockholm University, S-10691 Stockholm, Sweden.

* These authors equally contributed to this work. Correspondence and requests for materials should be addressed to S.C. (email: chesa@sjtu.edu.cn).
} 
T he optical activity $(\mathrm{OA})$ of materials arises from the ability of a medium to have different interaction for left and right circularized light ${ }^{1-6}$. Various chiral inorganic materials with scattering-, refraction- and absorption-based OA have been investigated extensively. Scattering at the inorganic/air interfaces of the helical arrays, such as for chiral films of $\mathrm{MgF}_{2}, \mathrm{TiO}_{2}$ and $\mathrm{SiO}_{2}$, tends to transmit circularly polarized light of one handedness and reflect the other in a wavelength, which depends on the pitch length and effective refractive index ${ }^{1,2,7-9}$; while refraction by the helical arrangement of the basic building blocks, such as chiral crystals of quartz, $\mathrm{NaClO}_{3}$ and $\mathrm{NaBrO}_{3}$, results in different velocities of right- and left-handed circularly polarized light in the medium ${ }^{3}$. These scattering-based OA and refraction-based OA are related to the geometry and refractive index regardless of the type of inorganics. For the absorptionbased OA inorganic materials, although inorganic nanoparticles functionalized with chiral organic molecules and assembled helically in organic assemblies gave rise to $\mathrm{OA}$ in their visible absorption region, such $\mathrm{OA}$ is due to plasmon oscillations of the excited electron in the given metal nanoparticles, and disappeared after removing the organics ${ }^{10-12}$. Therefore, it is still a challenge to explore new types of optically active inorganic materials featuring optical, electrical, mechanical and biological properties.

As known, molecular electronic transitions in organic and inorganic compounds occur when electrons are excited from a lower energy level to a higher energy level, which results in absorption of the electromagnetic wave with the corresponding energy difference between the two levels covering a wide energy range. In organic materials that have a helical geometry, the electronic transition-based optical activity (ETOA) at the absorption bands due to electronic exciton coupling is ubiquitous. However, although many inorganic materials exhibit electronic transitions in the ultraviolet or visible range of the electromagnetic spectrum, no electronic transition-based optically active inorganic materials have been reported up to now. Several macroscopic helical morphologies of inorganic materials were successfully synthesized by templating methods to transcribe organic molecules or assemblies onto inorganic materials (for example, semiconductors ${ }^{13,14}$, metal oxides ${ }^{15}$, carbonates $^{16}$ and mesoporous silica materials ${ }^{2,17,18}$ ), which might have molecular chirality with OA similar to organics. However, in contrast to organic materials, all of these materials do not result in $\mathrm{OA}$ at the absorption bands, probably because either non-photosensitive inorganics have been used or large pitch length has been formed.

In this investigation, $\mathrm{TiO}_{2}$ was used to study the ETOA of inorganic materials, as the anatase form typically exhibits ultraviolet absorption in the region of $200-400 \mathrm{~nm}$. We synthesized chiral $\mathrm{TiO}_{2}$ with a pitch length shorter than $100 \mathrm{~nm}$ via a templating method to transcribe the helical structure of lipid fibres onto the microscopic helical assembly of inorganic $\mathrm{TiO}_{2}$. Lipid amphiphilic molecules of $\mathrm{N}$-acylamino acids with hydrophobic chains were used as a template, which can self-organize to form helical ribbons or fibres with a bilayer molecular arrangement ${ }^{19}$. In particular, titanium diisopropoxide bis(acetylacetonate) (TDA) was used as the $\mathrm{TiO}_{2}$ source to achieve full interaction between template and inorganic species for effective transcription by decreasing condensation rate.

In our previous work, the helical lipid aggregation of $\mathrm{N}$-acyl-Lglutamic acid $\left(\mathrm{C}_{n}\right.$-L-Glu) was shown to template chiral mesoporous poly(pyrrole) with a short pitch length of $100 \mathrm{~nm}$ through fine electrostatic interactions between the negatively charged head groups of the lipid amphiphilic molecules and the positively charged imine nitrogens of poly(pyrrole $)^{19}$. Decreasing the repulsion between the negatively charged carboxylate head groups to allow closer interactions was the key to the formation of a short pitch length.
The strategy used in this investigation to synthesize chiral $\mathrm{TiO}_{2}$ with a short pitch length was to control the interaction between the carboxylic head groups of $\mathrm{C}_{n}-\mathrm{L} / \mathrm{D}-\mathrm{Glu}$ and the $\mathrm{TiO}_{2}$ species. Conventional $\mathrm{TiO}_{2}$ sources are generally considered to be inappropriate for the synthesis of $\mathrm{TiO}_{2}$ fibres because the hydrolysis rates of these titanium salts and alkoxides, as well as the condensation rates of the highly reactive $\mathrm{M}-\mathrm{OH}$ species, are too rapid. The high rates lead to continuous/homogeneous nucleation and growth, and the $\mathrm{TiO}_{2}$ never interacts with other ligands. Thus, organic templating is extremely difficult. If a slow, controlled nucleation can be driven and fine interactions with carboxylic acids maintained, then $\mathrm{TiO}_{2}$ fibres can be templated by carboxylic acid amphiphilic molecules. The coordination bonding would allow closer interactions between carboxylate head groups similar to the formation of mesoporous poly(pyrrole), and thus resulted in short pitch length ${ }^{19}$. The hydrolysis of TDA occurs slowly in the $\mathrm{pH}$ range of 3-8. The synthetic system used in this report relied upon the use of TDA to decrease the rate of the hydrolysis and condensation reactions of the $\mathrm{TiO}_{2}$ source. Additionally, interactions between lipid helical fibres through the titanium-carboxylic acid (Ti-COOH) coordination at the interface would result in the formation of lipid- $\mathrm{TiO}_{2}$ hybrid helical fibres. Through calcination, the organic lipid can be subsequently removed, and the amorphous $\mathrm{TiO}_{2}$ can be transformed to a pure inorganic crystalline phase.

Here, the synthesis of chiral $\mathrm{TiO}_{2}$ fibres was achieved via transcription of the helical structure of amino acid-derived amphiphile fibres through fine coordination bonding interactions between the carboxylic groups and the $\mathrm{TiO}_{2}$ source. The amorphous and crystal $\mathrm{TiO}_{2}$ double-helical fibres exhibited optical response to circularly polarized light at their absorption bands, which was attributed to the semiconductor $\mathrm{TiO}_{2}$-based electronic transitions from the valence band to the conduction band under an asymmetric electric field.

\section{Results}

Structure of chiral lipid- $\mathrm{TiO}_{2}$ hybrid nanofibres. Antipodal lipid- $\mathrm{TiO}_{2}$ hybrids with a pale yellow colour were synthesized using $\mathrm{N}$-stearoyl-L/D-glutamic acid $\left(\mathrm{C}_{18}\right.$-L/D-Glu) lipid enantiomers as the template and TDA as the $\mathrm{TiO}_{2}$ source in a methanol/ water solution (see the Methods section). As shown in Fig. 1a and b, scanning electron microscopy (SEM) revealed that the asprepared lipid- $\mathrm{TiO}_{2}$ hybrid that was synthesized with $\mathrm{C}_{18}$-D-Glu was exclusively composed of right-handed double-helical fibres. The width of a single fibre was $\sim 25 \mathrm{~nm}$. From the distinct helical morphology, the pitch along the axis of the fibre was estimated to be $\sim 100 \mathrm{~nm}$, which is indicated by the distance between the two black (or white) arrows. The $\mathrm{C}_{18}$-L-Glu molecule gave mirrorimage profiles with the same size and quality (Supplementary Fig. 1). The as-prepared left- and right-handed lipid- $-\mathrm{TiO}_{2}$ hybrid fibres are denoted as $\mathrm{L}-\mathrm{TiO}_{2}$-as and $\mathrm{R}-\mathrm{TiO}_{2}$-as, respectively.

High-resolution transmission electron microscopy (HRTEM) image (Fig. 1c) indicated that the fibres possess inner tubes with a diameter of $\sim 12 \mathrm{~nm}$ that are constant along the central axis of each individual tube. All of the helical tubes were open-ended. The $\mathrm{N}_{2}$ adsorption-desorption results showed that no pores corresponding to the size observed in the transmission electron microscopy (TEM) image existed in the lipid $-\mathrm{TiO}_{2}$ hybrid, which indicated that the inner tubes were filled with the lipid (Supplementary Fig. 2). Similar to the chiral mesoporous poly (pyrrole) reported in our previous work ${ }^{19}$, the helical tube was probably the self-assembled, lamellar-structured $\mathrm{C}_{18}$-D-Glu rectangular lipid used to template the helical $\mathrm{TiO}_{2}$. At first, the carboxylic acid group of the $\mathrm{C}_{18}$-D-Glu interacted with the titanium through coordination bonding, and the $\mathrm{TiO}_{2}$ began 

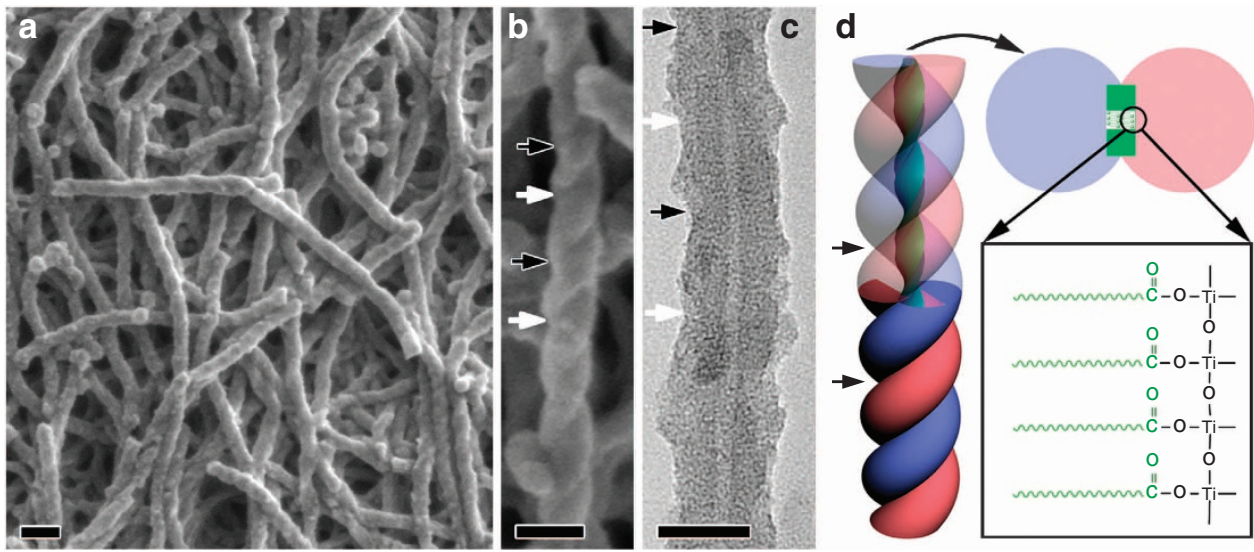

Figure 1 | Microscopy and schematic drawings of the as-prepared organic lipid-TiO $\mathbf{z}_{\mathbf{2}}$ hybrid fibres. SEM images, TEM images and schematic drawings of the as-prepared organic lipid- $\mathrm{TiO}_{2}$ hybrid fibres synthesized with $\mathrm{C}_{18}$-D-Glu $\left(\mathrm{R}-\mathrm{TiO}_{2}\right.$-as). The molar composition for the synthesis was $\mathrm{C}_{18}$-D-Glu:TDA:MeOH: $\mathrm{H}_{2} \mathrm{O}=1: 7.5: 5351: 55962$. (a,b) SEM images with different magnifications that show the right-handed double-helical microscopic features of the fibres with a pitch length of $\sim 100 \mathrm{~nm}$, as indicated by the distance between the two black (or white) arrow heads (JEOL JSM-7401F). The samples were observed without any metal coating. (c) TEM image that shows a tube in the centre of the fibre. Images were acquired using a JEOL JEM2100 TEM at $200 \mathrm{kV}$. (d) Schematic drawing of a structural model of the chiral $\mathrm{TiO}_{2}$ tubular fibre, which shows the lamellar structure of the $\mathrm{C}_{18} \mathrm{~B}^{-\mathrm{D}-\mathrm{Glu}}$ rectangular lipid fibre template for the formation of the lipid- $\mathrm{TiO}_{2}$ hybrid through fine coordination interaction between the carboxylic acid and titanium. The scale bars in $\mathbf{a}, \mathbf{b}$ and $\mathbf{c}$ represent 100,50 and $10 \mathrm{~nm}$, respectively.

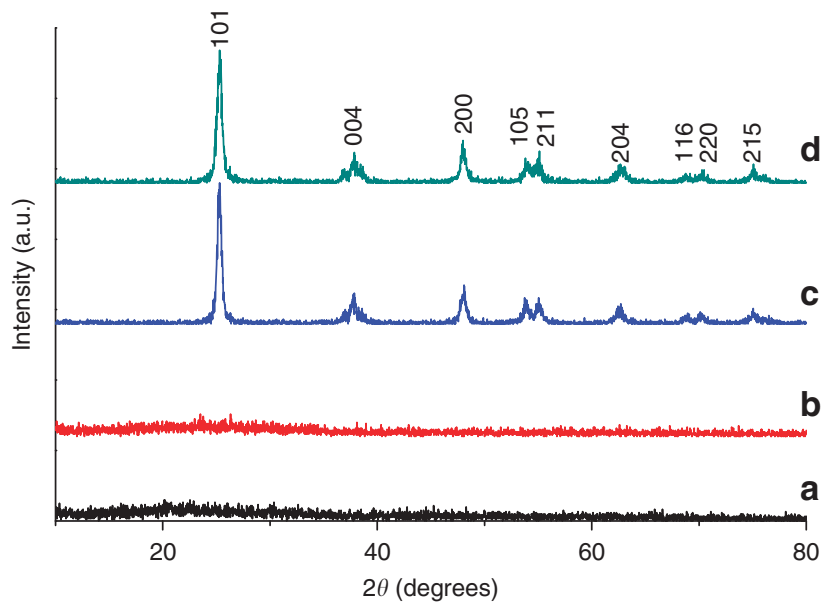

Figure 2 | X-ray diffraction patterns of as-prepared and calcined chiral $\mathrm{TiO}_{\mathbf{2}}$ fibres. X-ray diffraction patterns of as-prepared ((a) $\mathrm{L}_{-} \mathrm{TiO}_{2}$-as and (b) $\mathrm{R}$ - $\mathrm{TiO}_{2}$-as) and calcined ((c) $\mathrm{L}-\mathrm{TiO}_{2}$-cal and (d) $\mathrm{R}-\mathrm{TiO}_{2}$-cal) chiral

$\mathrm{TiO}_{2}$ fibres, which indicate that the as-prepared samples were amorphous. The $\mathrm{TiO}_{2}$ obtained after calcination at $550^{\circ} \mathrm{C}$ was assigned as the anatase phase, and there was no indication of other crystalline by-products.

templating the helical ribbon, which subsequently induced the formation of $\mathrm{a} \mathrm{TiO}_{2}$ wall. The preferential interaction with both large sides of the rectangular tape led to the formation of doublehelical $\mathrm{TiO}_{2}$, as shown in Fig. 1d. The presence of coordination bonding was confirmed by Fourier transform infrared spectroscopy (Supplementary Fig. 3).

Structure of calcined chiral $\mathrm{TiO}_{2}$ nanofibres. The as-prepared amorphous lipid- $\mathrm{TiO}_{2}$ hybrids were converted to white crystalline $\mathrm{TiO}_{2}$ by calcination in air at elevated temperatures. The X-ray diffraction patterns (Fig. 2) showed that the as-prepared fibres were amorphous in structure with essentially no diffraction peaks. However, the $\mathrm{TiO}_{2}$ obtained after calcination at $550{ }^{\circ} \mathrm{C}$ was assigned to the anatase phase (JCPDS File No. 21-1272) without any indication of other crystalline by-products. As can be observed in the SEM image (Fig. 3a) of the calcined sample, although the fibres no longer had a smooth surface, the righthanded double-helical morphology remained. The fibres were composed of stacked $\mathrm{TiO}_{2}$ nanoparticles with an average diameter of $\sim 20 \mathrm{~nm}$, which indicates that an obvious shrinkage occurred during calcination without destruction of the helical structure. The calcined left- and right-handed $\mathrm{TiO}_{2}$ fibres are denoted as $\mathrm{L}-\mathrm{TiO}_{2}$-cal and $\mathrm{R}-\mathrm{TiO}_{2}$-cal, respectively.

The HRTEM image shown in Fig. $3 \mathrm{~b}$ revealed that the calcined $\mathrm{TiO}_{2}$ fibres were composed of stacks of small anatase single crystals with an average size of $\sim 20 \mathrm{~nm}$ along the long axis of the fibre. It is worthy to note that the SEM image and the adsorption contrast of the TEM image indicate that the calcined fibres generally retained the double-helical morphology of the as-prepared samples. However, the former hollow tubular structures disappeared upon the growth of the anatase nanocrystals. Interestingly, the HRTEM image shows that an array of nanocrystals exhibits the contrast close to $\langle 111\rangle$ zone axis of the anatase structure, which indicates that the nanocrystals must have a preferential growth direction during the crystallization process. Besides, a twin boundary, formed by two anatase nanocrystals sharing their common (01) facets, can be clearly observed, as indicated by the white arrow heads. Additionally, it has been observed that the $\{101\}$ facets of the nanocrystals were often exposed, as indicated by the black arrows in the image. The morphology of anatase crystals are in good agreement with early experimental observations and theoretical calculations, in which the morphologies were dominated by the thermodynamically stable $\{101\}$ facets (constitutes more than $94 \%$ of the crystal surface according to the Wulff construction) and $\{001\}$ facets $^{20}$. In conclusion, the nanocrystals are formed according to the initially formed double-helical structure and the crystallization to anatase. The double-helical morphology caused the nanocrystals to maintain a chiral packing relationship. Therefore, a possible structural model is established, as shown in Fig. 3c. In particular, the adjacent nanocrystals have a rotational misplaced arrangement, piling up by sharing one of their $\{101\}$ facets while keeping one of the $\langle 111\rangle$ axis in common, leading to a helical array of nanocrystals with almost parallel $\langle 111\rangle$ axes. 

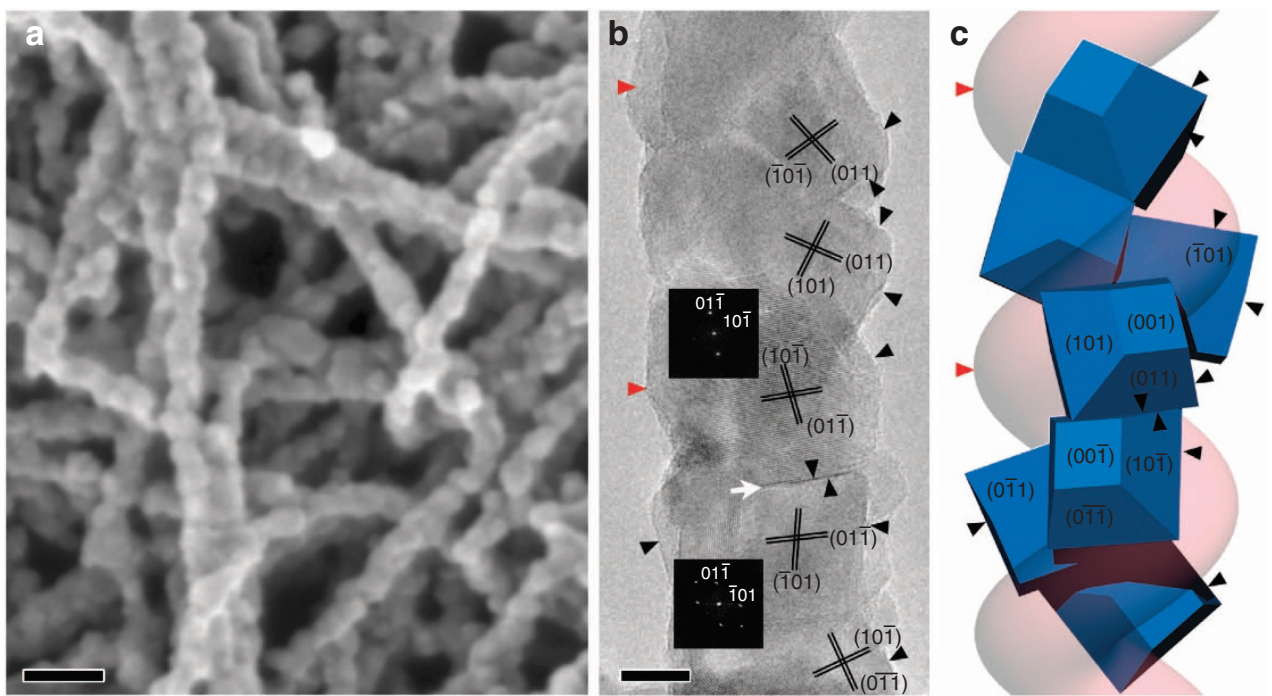

Figure 3 | Electron microscopy and schematic drawing of calcined chiral crystalline $\mathrm{TiO}_{2}$. (a) SEM image that shows the right-handed helical microscopic features of the sample (JEOL JSM-7401F). (b) The HRTEM image and the corresponding Fourier diffractograms of two nanocrystals. (c) Structural model showing the helical stacking of $\mathrm{TiO}_{2}$ anatase nanocrystals and good correspondence with the double-helical morphology. The common facets of the nanocrystals are $(0 \overline{1} 1),(\overline{1} 01),(\overline{1} 0 \overline{1}),(01 \overline{1}),(\overline{1} 01),(0 \overline{1})$ from top to bottom, respectively. The other strand of the double-helix fibre (pink) is shown without crystal particles. The scale bars in $\mathbf{a}$ and $\mathbf{b}$ represent 50 and $10 \mathrm{~nm}$, respectively.

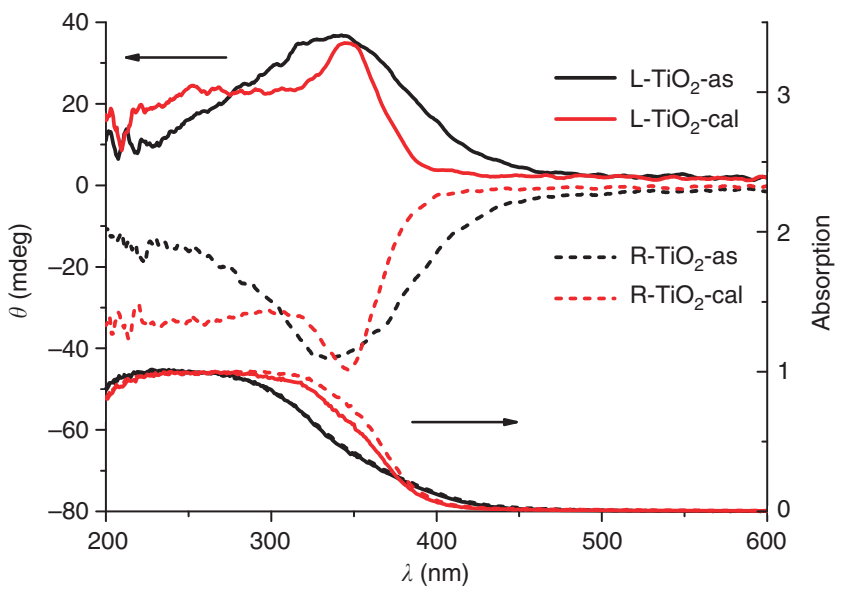

Figure 4 | Ultraviolet-vis and DRCD spectra of antipodal as-prepared and calcined chiral $\mathrm{TiO}_{2}$ fibres. Ultraviolet-vis and DRCD spectra of antipodal as-prepared $\left(\mathrm{L}-\mathrm{TiO}_{2}\right.$-as and $\mathrm{R}-\mathrm{TiO}_{2}$-as) and calcined $\left(\mathrm{L}-\mathrm{TiO}_{2}\right.$-cal and $\mathrm{R}-\mathrm{TiO}_{2}$-cal, $550^{\circ} \mathrm{C}$ ) chiral $\mathrm{TiO}_{2}$ fibres that show the ultraviolet absorption features and mirror-image $\mathrm{CD}$ signals that occurred at the $\mathrm{TiO}_{2}$ semiconductor absorption band.

Both the lattice planes and the morphologies of the nanocrystals fit the HRTEM image perfectly (marked by the black arrow heads). However, the contrast-overlap problem observed in the HRTEM image and the small crystal size caused the determination of the structural relationship of all the nanocrystals to be extremely difficult. Even so, it can be deduced that both helical strands have the similar epitaxial relationship and the $\mathrm{TiO}_{2}$ fibres generally still possess the double-helix chiral packing feature. Further investigation is still under way.

OA of chiral lipid- $\mathrm{TiO}_{2}$ hybrid and pure $\mathrm{TiO}_{2}$ nanofibres. The chiral configuration of the amorphous $\mathrm{TiO}_{2}$ and the helical stacking of the $\mathrm{TiO}_{2}$ nanocrystals endowed the materials with
OA, which was unambiguously detected by diffused reflection circular dichroism (DRCD). Figure 4 shows the DRCD and ultraviolet-visible (ultraviolet-vis) spectra of two pairs of antipodal lipid- $\mathrm{TiO}_{2}$ hybrid and calcined $\mathrm{TiO}_{2}$. The ultraviolet-vis spectra of the four samples each exhibited a broad absorption band in the range of $200-400 \mathrm{~nm}$, which was attributed to the electronic transition from the valence band to the conduction band of the $\mathrm{TiO}_{2}$ semiconductor. Compared with the crystalline anatase, the amorphous $\mathrm{TiO}_{2}$ consisted of crystallites smaller than $50 \AA$ in diameter with electronic properties that differed from those of the bulk materials ${ }^{21}$. The absorption of the as-prepared lipid- $\mathrm{TiO}_{2}$ exhibited a slight hypsochromic shift compared with the calcined lipid- $\mathrm{TiO}_{2}$, which was most likely due to the quantum size effect of semiconductor crystallites ${ }^{21}$. The yellowcolour and the long-tail absorption at wavelengths greater than $400 \mathrm{~nm}$ were attributed to narrowing of the band gap in the electronic structure due to the presence of organics on the $\mathrm{TiO}_{2}$ (ref. 22). The antipodal left- and right-handed amorphous $\mathrm{TiO}_{2}$ showed strong mirror-image critical dimension (CD) signals with a peak at $\sim 335 \mathrm{~nm}$ corresponding to the absorption edges, which indicates that the $\mathrm{OA}$ of the chiral $\mathrm{TiO}_{2}$ was primarily a reflection of the intrinsic electronic structure. These samples selectively reflect left- or right-handed circularly polarized light in the ultraviolet absorption region through a vicinal effect of helically arranged $\mathrm{TiO}_{2}$ species. As expected, a slight bathochromic shift was observed in the $C D$ signals $(\sim 350 \mathrm{~nm})$ for the calcined anatase samples in the corresponding absorption region, which indicates that the helical stacking of $\mathrm{TiO}_{2}$ crystals that were in close proximity induced strong $\mathrm{CD}$ signals. On the basis of structural considerations, it can be considered that the dynamic Coulomb interaction under dissymmetric field electron transition from the valence band to the conduction band of $\mathrm{TiO}_{2}$ results in its $\mathrm{OA}^{23,24}$. As known, the interaction between excited states of chromophores in chiral environments, called the exciton coupling, often give a split bisignate $\mathrm{CD}$ curves with the zerocrossing at the maximum absorption ${ }^{25,26}$. However, such exciton coupling spectra effect was not observed in the chiral $\mathrm{TiO}_{2}$ material. The reason for it may be that inorganic such as metals and metal oxides always have a manifold of electronic transitions 


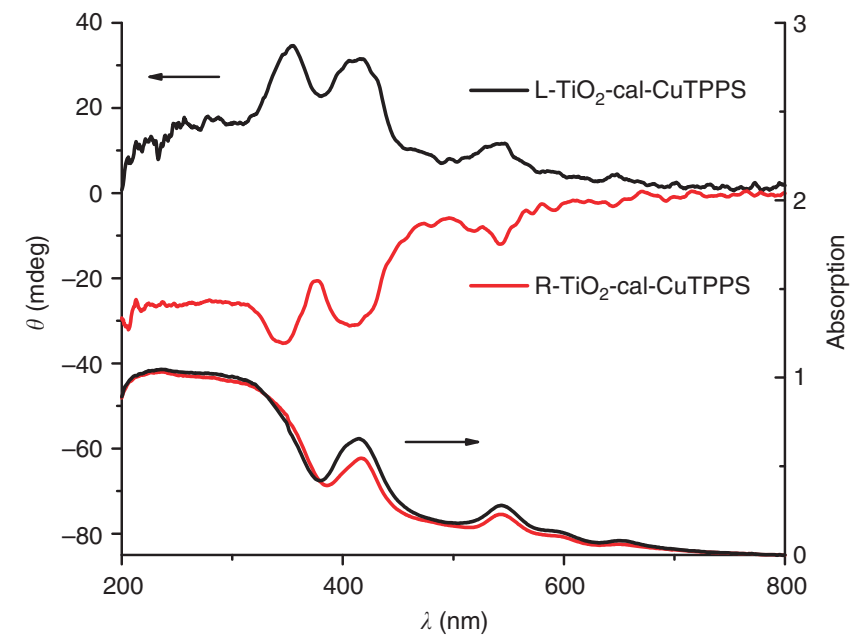

Figure 5 | Ultraviolet-vis and DRCD spectra of CuTPPS assembled on calcined chiral $\mathrm{TiO}_{2}$ fibres. Ultraviolet-vis and DRCD spectra of CuTPPS assembled directly on the calcined $\left(\mathrm{L}-\mathrm{TiO}_{2}\right.$-cal and $\mathrm{R}-\mathrm{TiO}_{2}$-cal, $550{ }^{\circ} \mathrm{C}$ ) chiral $\mathrm{TiO}_{2}$ fibres that show the ultraviolet absorption features and mirrorimage CD signals of the chiral $\mathrm{TiO}_{2}$-CuTPPS complex.

and, thus, the electronic transition-based circular dichroism may contain multiple absorption bands resulting in their overlaping ${ }^{27}$.

Dual OA by induced chiral assembly. Such long-range anisotropy of $\mathrm{TiO}_{2}$ could be expected to direct the epitaxial selfassembly of the guest molecules. Porphyrins were selected as example, because they have aroused much popular interest for the inherent electronic and optical properties. Figure 5 shows the DRCD and ultraviolet-vis spectra of copper (II) complexed tetra4-sulfonatophenyl-porphyrin (CuTPPS) assembled on the chiral $\mathrm{TiO}_{2}$ at $\mathrm{pH}=7.4$. The ultraviolet-vis spectra of both complexes show a broad Soret band with $417 \mathrm{~nm}$ and four weak Q bands were well resolved in the region of $500-700 \mathrm{~nm}$. However, no obvious blue or red shift of these bands was observed to support the existence of an $\mathrm{H}$ or J aggregate. It showed that the CuTPPS disks are almost monodispersed on the chiral $\mathrm{TiO}_{2}$ with a weak coupling effect of the conjugated ring systems. Nevertheless, a pair of mirror-image DRCD spectra at the Soret and Q bands indicates that CuTPPS was helically stacked on the surface of $\mathrm{TiO}_{2}$ mesocrystals due to helical arrangement of individual crystal of the chiral $\mathrm{TiO}_{2}$ (ref. 28). Such a $\mathrm{TiO}_{2} /$ CuTPPS complex is worth expecting to be used in dye-sensitized solar cells, photocatalysts, and so on, because that the unique arrangement of photosensitizer on semiconductor materials with the coupling effect could facilitated electron transfer and reduce recombination of the photogenerated electron-hole pairs arising from electronic interactions at their interface ${ }^{29-31}$.

\section{Discussion}

To the best of our knowledge, this is the first synthesis of an inorganic material with $\mathrm{OA}$ that has attributed to the electronic transition, which differs from optically active inorganic materials in which the $\mathrm{OA}$ is induced from the scattering or refraction of light and metal plasmonic oscillations. ETOA materials exhibit $\mathrm{OA}$ in the wavelength region that depends on the inorganic materials and their self-electronic state; therefore, it is possible to control the wavelength of the $\mathrm{OA}$ in the full ultraviolet-vis region by using different inorganic compounds. Such chiral materials have a clear advantage over both chiral organic assemblies and chiral nanoparticles supported by organics due to their increased physical robustness, which yields a permanent molecular inorganic chiral property. Microscopic chirality in inorganic materials could present both daunting challenges and significant opportunities in various research areas, such as physics, chemistry, material science, bioscience, devices (for example, optical, electrical, and mechanical), and the origin of life.

\section{Methods \\ Preparation of optically active helical $\mathrm{TiO}_{2}$ nanofibres. The helical $\mathrm{TiO}_{2}$ nanofibres can be synthesized over a wide range of molar compositions of $\mathrm{C}_{18^{-}}$- Glu:TDA:MeOH: $\mathrm{H}_{2} \mathrm{O}=1: x: y: 55962$, where $x$ and $y$ can be adjusted in the range of 5-60 and 3,800-6,600, respectively. A temperature of $45-60^{\circ} \mathrm{C}$ is favourable for the formation of helical fibres. In a typical synthesis, $\mathrm{C}_{18}$-D-Glu $(0.03 \mathrm{~g}, 0.08 \mathrm{mmol})$ was dissolved in a mixture of methanol $(13.6 \mathrm{~g})$ and deionized water $(80 \mathrm{ml})$ while stirring at room temperature. After the mixture was stirred for $10 \mathrm{~min}$, TDA $(0.29 \mathrm{~g}, 75 \%$ in isopropanol, purchased from TCI) was added to the mixture with stirring at $55^{\circ} \mathrm{C}$. The mixture was allowed to react at $55^{\circ} \mathrm{C}$ with stirring for $2 \mathrm{~h}$. The products were collected by centrifugal separation and dried by freeze drying at $-60{ }^{\circ} \mathrm{C}$, which resulted in a pale yellow powder. All organics in this product were removed by calcination at $550^{\circ} \mathrm{C}$, and crystalline anatase was obtained. The method for the synthesis of the helical $\mathrm{TiO}_{2}$ nanofibres using $\mathrm{C}_{18}$-L-Glu is the same as the above.}

Inducing self-assembly of CuTPPS on the chiral $\mathrm{TiO}_{2}$. CuTPPS ( $5 \mathrm{mg}$, purchased from TCI) was dissolved in $5 \mathrm{ml}$ of ion-exchanged water and a desired amount of aqueous $\mathrm{NaOH}$ solution $\left(0.1 \mathrm{mmoll}^{-1}\right)$ with stirring for $2 \mathrm{~min}$ at room temperature. The calcined $\mathrm{TiO}_{2}$ nanofibres $(50 \mathrm{mg}$ ) were then dispersed in the above solution with stirring for $2 \mathrm{~min}$, and then standing for $20 \mathrm{~min}$ at room temperature. The orange solid was separated with a centrifuge and dried by freeze drying at $-60^{\circ} \mathrm{C}$.

Characterization. The morphology of the chiral $\mathrm{TiO}_{2}$ nanofibres was observed with SEM (JEOL JSM-7401F) with an accelerating voltage of $1.0 \mathrm{kV}$. HRTEM images were taken with a JEOL JEM-2100 microscope operating at $200 \mathrm{kV}$. Powder $\mathrm{X}$-ray diffraction patterns were recorded on a Rigaku X-ray diffractometer D/ MAX-2200/PC equipped with $\mathrm{Cu} \mathrm{K} \alpha$ radiation $(40 \mathrm{kV}, 20 \mathrm{~mA})$. Ultraviolet and DRUV spectra were taken by using a Shimadzu UV-2450 spectropolarimeter fitted with DRUV apparatus. CD and DRCD spectra were taken by using a JASCO J-815 spectropolarimeter fitted with DRCD apparatus. The $\mathrm{N}_{2}$ adsorption/desorption isotherms were obtained at $77 \mathrm{~K}$ by using a Quantachrome NOVA $4200 \mathrm{E}$ surface area and pore-size analyser. Fourier transform infrared spectra were recorded on a Perkin-Elmer Paragon 1000 spectrometer with a resolution of $2 \mathrm{~cm}^{-1}$ using the $\mathrm{KBr}$ method.

\section{References}

1. Robbie, K., Broer, D. \& Brett, M. Chiral nematic order in liquid crystals imposed by an engineered inorganic nanostructure. Nature 399, 764-766 (1999).

2. Shopsowitz, K. E., Qi, H., Hamad, W. Y. \& MacLachlan, M. J. Free-standing mesoporous silica films with tunable chiral nematic structures. Nature 468, 422-425 (2010).

3. Moxon, J., Renshaw, A. \& Tebbutt, I. The simultaneous measurement of optical activity and circular dichroism in birefringent linearly dichroic crystal sections. II. Description of apparatus and results for quartz, nickel sulphate hexahydrate and benzil. J. Phys. D: Appl. Phys. 24, 1187-1192 (1991).

4. Bartus, J., Weng, D. \& Vogl, O. Optical activity measurements on solids. 6. Solid state optical activity and circular dichroism measurements of sodium thioantimonate nonahydrate. Monatsh. Chem. 125, 671-680 (1994).

5. Johnson, W., Berova, N., Nakanishi, K. \& Woody, R. Circular Dichroism: Principles and Applications. 2nd edn (Wiley-VCH, 2000).

6. Barron, L. D. Molecular Light Scattering and Optical Activity (Cambridge University Press, 2004).

7. van Popta, A. C., Sit, J. C. \& Brett, M. J. Optical properties of porous helical thin films. Appl. Opt. 43, 3632-3639 (2004).

8. Soukoulis, C. M. \& Wegener, M. Past achievements and future challenges in the development of three-dimensional photonic metamaterials. Nat. Photon 5, 523-530 (2011).

9. Hentschel, M., Schäferling, M., Weiss, T., Liu, N. \& Giessen, H. Threedimensional chiral plasmonic oligomers. Nano Lett. 12, 2542-2547 (2012).

10. Govorov, A. O. et al. Chiral nanoparticle assemblies: circular dichroism, plasmonic interactions, and exciton effects. J. Mater. Chem. 21, 16806-16818 (2011). 
11. Kuzyk, A. et al. DNA-based self-assembly of chiral plasmonic nanostructures with tailored optical response. Nature 483, 311-314 (2012).

12. Guerrero-Martínez, A., Alonso-Gómez, J. L., Auguié, B., Cid, M. M. \& LizMarzán, L. M. From individual to collective chirality in metal nanoparticles. Nano Today 6, 381-400 (2011).

13. Sone, E. D., Zubarev, E. R. \& Stupp, S. I. Semiconductor nanohelices templated by supramolecular ribbons. Angew. Chem. Int. Ed. 41, 1705-1709 (2002).

14. Van Bommel, K. J. C., Friggeri, A. \& Shinkai, S. Organic templates for the generation of inorganic materials. Angew. Chem. Int. Ed. 42, 980-999 (2003).

15. Kobayashi, S. et al. Preparation of helical transition-metal oxide tubes using organogelators as structure-directing agents. J. Am. Chem. Soc 124, 6550-6551 (2002).

16. Orme, C. et al. Formation of chiral morphologies through selective binding of amino acids to calcite surface steps. Nature 411, 775-779 (2001).

17. Che, S. et al. Synthesis and characterization of chiral mesoporous silica. Nature 429, 281-284 (2004).

18. Jung, J. H., Kobayashi, H., Masuda, M., Shimizu, T. \& Shinkai, S. Helical ribbon aggregate composed of a crown-appended cholesterol derivative which acts as an amphiphilic gelator of organic solvents and as a template for chiral silica transcription. J. Am. Chem. Soc 123, 8785-8789 (2001).

19. Fan, C. et al. Formation of chiral mesopores in conducting polymers by chiral-lipid-ribbon templating and "seeding" route. Adv. Funct. Mater 18, 2699-2707 (2008).

20. Lazzeri, M., Vittadini, A. \& Selloni, A. Structure and energetics of stoichiometric $\mathrm{TiO}_{2}$ anatase surfaces. Phys. Rev. B 63, 155409-155418 (2001).

21. Brus, L. E. Electron-electron and electron-hole interactions in small semiconductor crystallites: The size dependence of the lowest excited electronic state. J. Chem. Phys 80, 4403 (1984).

22. Chen, X. \& Mao, S. S. Titanium dioxide nanomaterials: Synthesis, properties, modifications, and applications. Chem. Rev. 107, 2891-2959 (2007).

23. Kuzyk, A. et al. DNA-based self-assembly of chiral plasmonic nanostructure with tailored optical response. Nature 483, 311-314 (2012).

24. Fan, Z. \& Govorov, A. O. Helical metal nanoparticle assemblies with defects: plasmonic chirality and circular dichroism. J. Phys. Chem. C 115, 13254-13261 (2011).

25. Kasha, M. Energy transfer mechanisms and the molecular exciton model for molecular aggregates. Radiat. Res 20, 55-70 (1963).

26. Harada, N. \& Nakanishi, K. Circular dichroic spectroscopy: exciton coupling in organic stereochemistry (University Science Books, Mill Valley, CA, 1983).

27. Pescitelli, G., Di Bari, L. \& Berova, N. Conformational aspects in the studies of organic compounds by electronic circular dichroism. Chem. Soc. Rev. 40, 4603-4625 (2011)
28. Qiu, H., Inoue, Y. \& Che, S. Supramolecular chiral transcription and recognition by mesoporous silica prepared by chiral imprinting of a helical micelle. Angew. Chem. Int. Ed 48, 3069-3072 (2009).

29. Patil, A. J., Lee, Y. C., Yang, J. W. \& Mann, S. Mesoscale integration in Titania/ J-Aggregate hybrid nanofibers. Angew. Chem. Int. Ed. 124, 757-761 (2012).

30. Fujii, Y., Tsukahara, Y. \& Wada, Y. pH-dependent reversible switching of fluorescence of water-soluble porphyrin adsorbed on mesoporous $\mathrm{TiO}_{2}$ film. Bull. Chem. Soc. Jpn. 79, 561-568 (2006).

31. Linsebigler, A. L., Lu, G. \& Yates, Jr J. T. Photocatalysis on $\mathrm{TiO}_{2}$ surfaces: principles, mechanisms, and selected results. Chem. Rev. 95, 735-758 (1995).

\section{Acknowledgements}

This work was supported by the National Natural Science Foundation of China (Gran No. 20890121), the 973 project (2009CB930403) of China, WCU (R-31-2008-000-100550, Korea) and Berzelii EXSELENT (Sweden). We thank Instrumental Analysis Centre of Shanghai Jiao Tong University for their collaboration on CD measurements.

\section{Author contributions}

S.C. designed and lead the project. S.L. synthesized helical $\mathrm{TiO}_{2}$ and carried out the low magnification SEM and CD measurements. L.H. processed the HRTEM observation and structural analysis. Y.D. contributed to CD analysis. S.A. and O.T. contributed to take high magnification SEM images and O.T. to discuss HRTEM characterization. Y.C., B.L., L.M. and J.Z. contributed to revision of this manuscript. S.C., L.H., Y.D. and S.L prepared the manuscript and contributed to the mechanism analysis. S.C. guided all aspects of the work.

\section{Additional information}

Supplementary Information accompanies this paper on http://www.nature.com/ naturecommunications

Competing financial interests: The authors declare no competing financial interests.

Reprints and permission information is available online at http://npg.nature.com/ reprintsandpermissions/

How to cite this article: Liu, S. et al. Synthesis of chiral $\mathrm{TiO}_{2}$ nanofibre with electron transition-based optical activity. Nat. Commun. 3:1215 doi: 10.1038/ncomms2215 (2012)

License: This work is licensed under a Creative Commons Attribution-NonCommercialShare Alike 3.0 Unported License. To view a copy of this license, visit http://creativecommons.org/licenses/by-nc-sa/3.0/ 